\title{
Hypoxia increases the heterogeneity of melanoma cell populations and affects the response to vemurafenib
}

\author{
DANIELA PUCCIARELLI ${ }^{1}$, NINA LENGGER ${ }^{1}$, MARTINA TAKÁČOVÁ $^{2}$, LUCIA CSADEROVA $^{2}$, \\ MARIA BARTOSOVA ${ }^{2}$, HEIMO BREITENEDER ${ }^{1}$, SILVIA PASTOREKOVA ${ }^{2}$ and CHRISTINE HAFNER ${ }^{1,3}$ \\ ${ }^{1}$ Department of Pathophysiology and Allergy Research, Center for Pathophysiology, Infectiology and Immunology, \\ Medical University of Vienna, Vienna A-1090, Austria; ${ }^{2}$ Institute of Virology, Department of Molecular Medicine, \\ Slovak Academy of Sciences, Bratislava 81101, Slovakia; ${ }^{3}$ Karl Landsteiner Gesellschaft, \\ Institute for Dermatological Research, St. Poelten A-3100, Austria
}

Received June 16, 2015; Accepted January 12, 2016

DOI: $10.3892 / \mathrm{mmr} .2016 .4888$

\begin{abstract}
A hypoxic microenvironment is one of the predominant reasons for incomplete response to melanoma treatment. Vemurafenib, which targets the mutated BRAF-V600 kinase, improves melanoma patient survival, however, resistance invariably develops. The present study evaluated the effect of hypoxia on three BRAF-V600E mutant melanoma cell lines, M14, A375 and 518A2, treated with vemurafenib. Compared with the other two cell lines, hypoxic vemurafenib-treated A375 cells exhibited an enhanced cell proliferation rate and migratory capacity compared with normoxic vemurafenib-treated A375 cells. Immunoblotting analyses revealed that the expression levels of hypoxia inducible factor (HIF) $1 \alpha$ and carbonic anhydrase IX were reduced in vemurafenib-treated M14 and 518A2 cells, however, not in A375 cells. The expression levels of the mitogen-activated protein kinase, Janus kinase-signal transducer and activator of transcription, and phosphatidylinositol-4,5-bisphosphate 3-kinase signaling pathway proteins revealed a cell-type specific response to vemurafenib and hypoxia. Knockdown experiments of HIFla performed in hypoxic A375 cells decreased the expression of phosphorylated (p-)protein kinase B, which was restored following vemurafenib treatment, and increased the expression of p-extracellular-signal-regulated kinases. Therefore, three melanoma cell lines responded to vemurafenib under hypoxia in a cell type-specific manner, suggesting that a subset of cells provides a treatment-resistant pool, from which disease
\end{abstract}

Correspondence to: Dr Christine Hafner, Department of Pathophysiology and Allergy Research, Center for Pathophysiology, Infectiology and Immunology, Medical University of Vienna, Waehringer Guertel 18-20, Vienna A-1090, Austria

E-mail: christine.hafner@meduniwien.ac.at

Abbreviations: HIF1 $\alpha$, hypoxia-inducible factor $1 \alpha$; CAIX, carbonic anhydrase IX

Key words: melanoma, hypoxia, HIF1 $\alpha$, CAIX, BRAF, vemurafenib relapse may originate. These data confirmed that vemurafenib may be successful in treating the proliferating cells, whereas the non-proliferating subpopulation must be addressed by a combination of vemurafenib with other treatment strategies.

\section{Introduction}

Hypoxia is a prominent feature of solid tumor types resulting in a broad range of effects on a number of cellular pathways and is one of the major contributors to the development of resistance to anticancer drugs (1). The limited supply of nutrition and oxygen creates hypoxic regions, which affect cancer progression and induce changes in cellular metabolism (2). Melanoma, one of the most aggressive and treatment-resistant solid tumor types, is also affected by hypoxia. The predominant transcriptional regulator of the hypoxic response is the heterodimeric hypoxia-inducible factor (HIF)1 (3), which serves an essential role in the maintenance of oxygen homeostasis (4). Oxygen levels affect protein stability, subcellular localization and transcriptional potency of the HIF1 $\alpha$ subunit. Under normoxic conditions, HIF1 $\alpha$ is hydroxylated on two conserved proline residues and is subsequently degraded via the proteasome (3). Hypoxia prevents the hydroxylation of HIFl $\alpha$, leading to its accumulation and translocation into the nucleus where it dimerizes with HIF1 $\beta$, and together they regulate the expression of hundreds of genes (3). One of these genes encodes carbonic anhydrase (CA)IX, which is involved in the regulation of $\mathrm{pH}$, tumor cell survival, adhesion and migration (2). CAIX exhibits only limited expression in normal tissues, however, its expression is highly elevated in various cancer types, including colorectal and lung carcinomas $(5,6)$. CAIX has not been shown to be expressed in melanomas (7). HIF1 is upregulated by mutated Ras and Braf, as well as by loss-of-function mutations of phosphatidylinositol-3,4,5-trisphosphate 3-phosphatase, and it has been reported that mutant BRAF-V600 increases the expression of HIF1 $\alpha$ in melanoma cells (8). BRAF is a key regulator of cell growth and proliferation in melanoma and when mutated can act as an oncogene (9).

Approximately $60 \%$ of melanomas display a mutation in the gene encoding the serine/threonine protein kinase, 
BRAF (10). The mutated protein can be inhibited by specific inhibitors (11). Vemurafenib, a specific BRAF-V600 kinase inhibitor, already in clinical use, arrests the cell-cycle and induces apoptosis in melanoma cells $(12,13)$. However, inhibition of BRAF often provides short and incomplete responses, followed by relapses (14). Hypoxia contributes to the relapse since it markedly influences the phenotype of melanoma cells, their aggressiveness and treatment sensitivity, leading to cancer progression. Hypoxia can trigger a dynamic, adaptive phenotypic response, where cells switch from a highly proliferative, poorly invasive phenotype to a highly invasive, less proliferative one, through changes in receptors involved in the non-canonical wingless-type MMTV integration site family, member 5A signaling pathway, tyrosine-protein kinase transmembrane receptor (ROR)1 and ROR2 (15).

The present study investigated the role of hypoxia on the treatment with vemurafenib in human melanoma cells. In a panel of different cell lines, hypoxia altered the metabolism and increased the cell motility of A375 cells exposed to vemurafenib. Further experiments revealed that vemurafenib had no affect on the protein expression levels of HIF1 $\alpha$ and CAIX in hypoxic A375 cells, suggesting a cell-type specific pattern of melanoma progression.

\section{Materials and methods}

Cell lines and reagents. The A375 human melanoma cell line was purchased from American Type Culture Collection (Rockville, MD, USA). The 518A2 and M14 melanoma cell lines were described previously (16). All cells were cultured in RPMI-1640 medium (Lonza, Inc., Verviers, Belgium), supplemented with $10 \%$ fetal calf serum (FCS; Gibco; Thermo Fisher Scientific, Inc., Waltham, MA, USA), at $37^{\circ} \mathrm{C}$ in a humidified $5 \% \mathrm{CO}_{2}$ incubator. Routine tests to exclude mycoplasma and to characterize the origin of the cells (short tandem repeat analysis) were performed. Genotyping for a BRAF-V600 mutation was performed using the BRAF StripAssay (ViennaLab, Vienna, Austria). Vemurafenib (PLX4032), a selective inhibitor of BRAF-V600, was purchased from Selleckchem (Houston, TX, USA). Exposure to hypoxia was performed in an anaerobic work station (Ruskin Technologies, Bridgend, UK) in $2 \% \mathrm{O}_{2}, 5 \% \mathrm{CO}_{2}, 10 \% \mathrm{H}_{2}$, and $83 \% \mathrm{~N}_{2}$, at $37^{\circ} \mathrm{C}$.

Real-time cell proliferation assays in normoxic and hypoxic conditions. In order to track the cell growth of melanoma cells treated with vemurafenib, a real-time characterization was performed using the xCELLigence system (ACEA Bioscience, Inc., San Diego, CA, USA). The impedance-based xCELLigence system ${ }^{\circledR}$ was placed at $37^{\circ} \mathrm{C}$ in a humidified $5 \% \mathrm{CO}_{2}$ incubator. A total of $5 \times 10^{3}$ melanoma cells/well were seeded and placed in the xCELLigence system ${ }^{\circledR}$. The proliferation was measured for $24 \mathrm{~h}$ prior to the addition of the following compounds: i) 1 or $5 \mu \mathrm{M}$ vemurafenib, ii) $1 \mathrm{mM}$ dimethyloxalylglycine (DMOG; Sigma-Aldrich, St. Louis, MO, USA), iii) 1 or $5 \mu \mathrm{M}$ vemurafenib plus $1 \mathrm{mM}$ DMOG. The plates were placed back in the $\mathrm{xCELLi-}$ gence system ${ }^{\circledR}$ and cell proliferation was measured for $100 \mathrm{~h}$. DMOG was used to induce hypoxia in cells when it was technically not possible to use a hypoxia chamber.
Determination of extracellular $\mathrm{pH}$ ( $\mathrm{pHe}$ ) and oxygen consumption in melanoma cells. Since hypoxia-induced changes in metabolism can affect pHe and oxygen consumption, these two parameters were measured by a non-invasive SDR optical sensor system (PreSens Precision Sensing GmbH, Regensburg, Germany) embedded in the hypoxic chamber. A total of $0.2 \times 10^{6}$ melanoma cells were seeded into 24-well OxoDish ${ }^{\circledR}$ plates (PreSens Precision Sensing GmbH) for the determination of oxygen consumption, and in 24-well HydroDish $^{\circledR}$ plates (PreSens Precision Sensing $\mathrm{GmbH}$ ) for the determination of $\mathrm{pHe}$, for $24 \mathrm{~h}$. The cells were subsequently incubated with $1 \mu \mathrm{M}$ vemurafenib. Oxygen and $\mathrm{pH}$ kinetics were visualized in real-time using the SDR optical sensor system. The pHe was measured every $25 \mathrm{~min}$ for $112 \mathrm{~h}$ and the oxygen consumption was measured every $5 \mathrm{~min}$ for $5 \mathrm{~h}$.

RNA extraction and reverse transcription-quantitative polymerase chain reaction ( $R T-q P C R)$. The total RNA was extracted using TRIzol reagent (Invitrogen; Thermo Fisher Scientific, Inc.,) from normoxic and hypoxic melanoma cells treated with vemurafenib $(1 \mu \mathrm{M})$. The RNA $(2 \mu \mathrm{g})$ was reverse-transcribed into cDNA using the High-Capacity cDNA Reverse Transcription Kit (Applied Biosystems, Foster City, CA, USA) using random heptameric primers. RT-qPCR analysis of HIFl $\alpha$ and $C A 9$, and $\beta$-actin as an internal standard, were performed on a StepOneTM Real-Time PCR system (Applied Biosystems) using the Power SYBR ${ }^{\circledR}$ Green PCR Master mix (Applied Biosystems). The primers used were as follows: HIFl $\alpha$, sense: 5'-GCT TGGTGCTGATTTGTGAACC-3' and antisense: 5'-GCA TCCTGTACTGTCCTGTGGTG-3'; CA9, sense: 5'-CCG AGCGACGCAGCCTTTGA-3' and antisense: 5'-GGC TCCAGTCTCGGCTACCT-3'; $\beta$-actin, sense: 5'-TCCTCC CTGGAGAAGAGCTA-3' and antisense: 5'-ACATCTGCT GGAAGGTGGAC-3'. The results were analyzed using the Applied Biosystems 7500 system v1.4.0 software (Applied Biosystems).

HIFla knockdown induced by small interfering (si)RNA in A375 melanoma cells. A total of $9 \times 10^{5}$ A 375 cells were plated into 6-well tissue culture plates, containing antibiotic-free growth medium. The cells were grown up to $60-80 \%$ confluence and were subsequently transfected with $H I F 1 \alpha$ specific siRNA oligonucleotides ( 7 and $10 \mu \mathrm{g} / \mathrm{ml}$; Santa Cruz Biotechnology, Inc., Santa Cruz, CA, USA) or with control siRNAs (7 and $10 \mu \mathrm{g} / \mathrm{ml}$ ) using the siRNA Reagent System (Santa Cruz Biotechnology, Inc.), according to the manufacturer's protocol. The transfected cells were grown in medium containing vemurafenib $(1 \mu \mathrm{M})$ for $24 \mathrm{~h}$, harvested and immunoblotting were performed.

Immunoblotting. A total of $1 \times 10^{5}$ melanoma cells were incubated with vemurafenib $(1 \mu \mathrm{M})$ and placed in normoxia or hypoxia for $24 \mathrm{~h}$. The cells were subsequently lysed in lysis buffer, as described previously (16), and equal quantities of protein (60 $\mu \mathrm{g}$ per lane) were separated on sodium dodecyl sulphate-polyacrylamide gel electrophoresis gels (Bio-Rad Laboratories, Inc., Hercules, CA, USA) under reducing conditions. The proteins were transferred onto polyvinylidene fluoride membranes (EMD Millipore, Billerica, MA, USA). 
and blocked with Tris-buffered saline (TBS; Gibco; Thermo Fisher Scientific, Inc.) supplemented with 3\% bovine serum albumin (BSA; Roth, Karlsruhe, Germany) for $2 \mathrm{~h}$ at room temperature. Subsequently, the membranes were incubated wit the following primary mouse anti-human immunoglobulin (Ig) $\mathrm{G}$ antibodies overnight at $4^{\circ} \mathrm{C}$ : Anti-HIF1 $\alpha$ (1:8,000; clone 54; 565924; BD Biosciences, San Jose, CA, USA), anti-CAIX (1:8,000; clone M75) (17), anti-phosphorylated (p-)focal adhesion kinase (FAK; Tyr397; 1:500; clone M121; ab24781), anti-FAK (1:5,000; clone 63D5; ab72140), anti-protein kinase C (PKC) $\alpha$ (1:1,000; clone M237; ab86715; all Abcam, Cambridge, UK), anti-p-protein kinase B (AKT; Ser473; 1:1,000; clone 587F11; 4051), anti-AKT (1:2,000; clone 40D4; 2920), anti-p-signal transducer and activator of transcription (STAT)3 (Tyr705; 1:2,000; clone M9C6; 4113), anti-STAT3 (1:1,000; clone 124H6; 9139), anti-p-extracellular-signal-regulated kinases (ERK)1/2 (Thr202/Tyr204; 1:2,000; clone E10; 9106), anti-ERK1/2 (1:2,000; clone $3 \mathrm{~A} 7$; 9107$)$ or anti- $\beta$-actin (1:1,000; clone 8H10D10; 3700; all Cell Signaling Technology, Inc., Danvers, MA, USA). Following washing three times for 5 min at room temperature with TBS supplemented with $0.5 \%$ BSA and $0.05 \%$ Tween 20 (Sigma-Aldrich), the membranes were incubated with corresponding peroxidase-conjugated horse anti-mouse $\mathrm{IgG}$ monoclonal secondary antibody (1:2,000; 7076; Cell Signaling Technology, Inc.) for $2 \mathrm{~h}$ at room temperature. Blots were developed with LumiGLO chemiluminescent substrate (Cell Signaling Technology, Inc.) and the bands were visualized using the Filmentwickler CP1000 Processor (AGFA, Mortsel, Belgium).

Wound-healing assay. To analyze 2D-cell migration behavior, a wound-healing assay was performed, as previously described (18). Melanoma cells seeded to confluence, were starved overnight in medium containing $0.5 \%$ FCS and treated with vemurafenib $(1 \mu \mathrm{M})$. A wound was made with a sterile micropipette tip. Images of the cells were captured immediately following wound initiation and then every $15 \mathrm{~min}$ for up to $48 \mathrm{~h}$ using an inverted Zeiss microscope (Axiovert40-CFL; Carl Zeiss, Oberkochen, Germany). Wound-healing was quantified using ImageJ software (version 2; National Institutes of Health, Bethesda, MA, USA) (19) and the results were analyzed by unpaired Student's t-test. $\mathrm{P}<0.05$ was considered to indicate a statistically significant difference.

\section{Results}

Hypoxia influences the cell growth of BRAF-V600E mutant melanoma cells treated with vemurafenib. All three melanoma cell lines tested harbor the BRAF-V600E mutation (data not shown). The influence of hypoxia on the cell growth of melanoma cell lines treated with vemurafenib was assessed in a real-time setting using the impedance-based xCELLigence system ${ }^{\circledR}$ for up to $140 \mathrm{~h}$ under normoxic and hypoxic conditions (Fig. 1A). Normoxic, vemurafenib-treated M14, A 375 and 518A2 cells exhibited reduced proliferation after $65 \mathrm{~h}$ by 67,80 and $20 \%$, respectively, compared with normoxic, untreated cells. At this time point, hypoxic M14 and A375 cells reduced their growth by 83 and $44 \%$, respectively, compared with the normoxic counterparts. The 518A2 cells were not susceptible to hypoxic conditions and the proliferation rate revealed no change. However, hypoxic vemurafenib-treated A375 cells exhibited an unexpected increase in the cell proliferation rate (cell index, 1) when compared with normoxic, vemurafenib-treated A375 cells (cell index, 0.5). Hypoxic, vemurafenib-treated M14 and 518A2 cells reduced growth by an additional 26 and 34, respectively, compared with normoxic, vemurafenib-treated M14 and 518A2 cells.

Modification of pHe and oxygen consumption in hypoxic, vemurafenib-treated melanoma cells. Cultivation of melanoma cells under hypoxia for $130 \mathrm{~h}$ in the presence of vemurafenib resulted in reduced acidification of the extracellular environment. Incubation with vemurafenib markedly reduced the $\mathrm{pHe}$ of the growth medium of hypoxic M14 cells. For the other two cell lines, pHe dropped at a reduced rate, reaching $6.3 \pm 0.02$ for A375 cells after $70 \mathrm{~h}$ and slightly increased to $6.5 \pm 0.02$. For 518A2 cells, the pHe dropped to $6.4 \pm 0.04$ after $75 \mathrm{~h}$ (Fig. 1B). No change was observed in oxygen consumption between treated and untreated 518A2 cells. A decrease of oxygen consumption was observed in M14 cells, whereas a slight increase was observed in the A375 cells (Fig. 1C).

Effect of hypoxia on HIFla and CAIX in vemurafenib-treated melanoma cells. Relative mRNA expression of HIFl $\alpha$ under hypoxic conditions were between 0.7 and 0.8 for all three cell lines. In normoxic M14 cells, the mRNA expression of HIFla was $1 \pm 0.3$ and it decreased following vemurafenib treatment under both hypoxic and normoxic conditions to $0.4 \pm 0.03$ and $0.2 \pm 0.07$, respectively. The relative mRNA expression of HIFla in normoxic A375 cells was $1 \pm 0.2$ and decreased to $0.7 \pm 0.2$ following vemurafenib treatment. However, under hypoxic conditions, vemurafenib treatment increased the mRNA expression of HIFl $\alpha$ to $1.4 \pm 0.4$ in A375 cells. The relative mRNA expression of $H I F 1 \alpha$ in 518A2 revealed no significant changes between the different treatment and culture conditions. The relative mRNA expression of $C A 9$ under normoxic conditions were between 1 and 2 in all three cell lines, and were reduced following treatment. The mRNA expression levels of $C A 9$ were $2.8 \pm 0.08$ for M14, 49.2 \pm 5.3 for A375 and $12.1 \pm 0.9$ for $518 \mathrm{~A} 2$ cell lines under hypoxia. Following treatment with vemurafenib, all three cell lines exhibited increased mRNA expression levels of $C A 9(5.8 \pm 0.7,90.0 \pm 2.7$ and 15.5 \pm 1.2 for M14, A375 and 518A2, respectively). All mRNA expression levels were normalized against the housekeeping gene coding for $\beta$-actin (Fig. 2A).

The protein expression levels of HIF1 $\alpha$ and CAIX were evaluated by immunoblotting. HIF1 $\alpha$ and CAIX were expressed in all three cell lines under hypoxia. However, the expression level of each protein was reduced in vemurafenib-treated M14 and 518A2 cells; this was not observed in A375 cells (Fig. 2B).

Hypoxia affects multiple signaling pathways in vemurafenib-treated melanoma cells. To further assess how hypoxia modifies the expression of proteins involved in cell growth, migration and survival, an extended analysis of signaling pathways by immunoblotting was performed. Melanoma cell lines were treated with vemurafenib and exposed to hypoxia or normoxia. Under hypoxia, the expression of p-FAK was downregulated in A375 and M14 cells, and was upregulated 

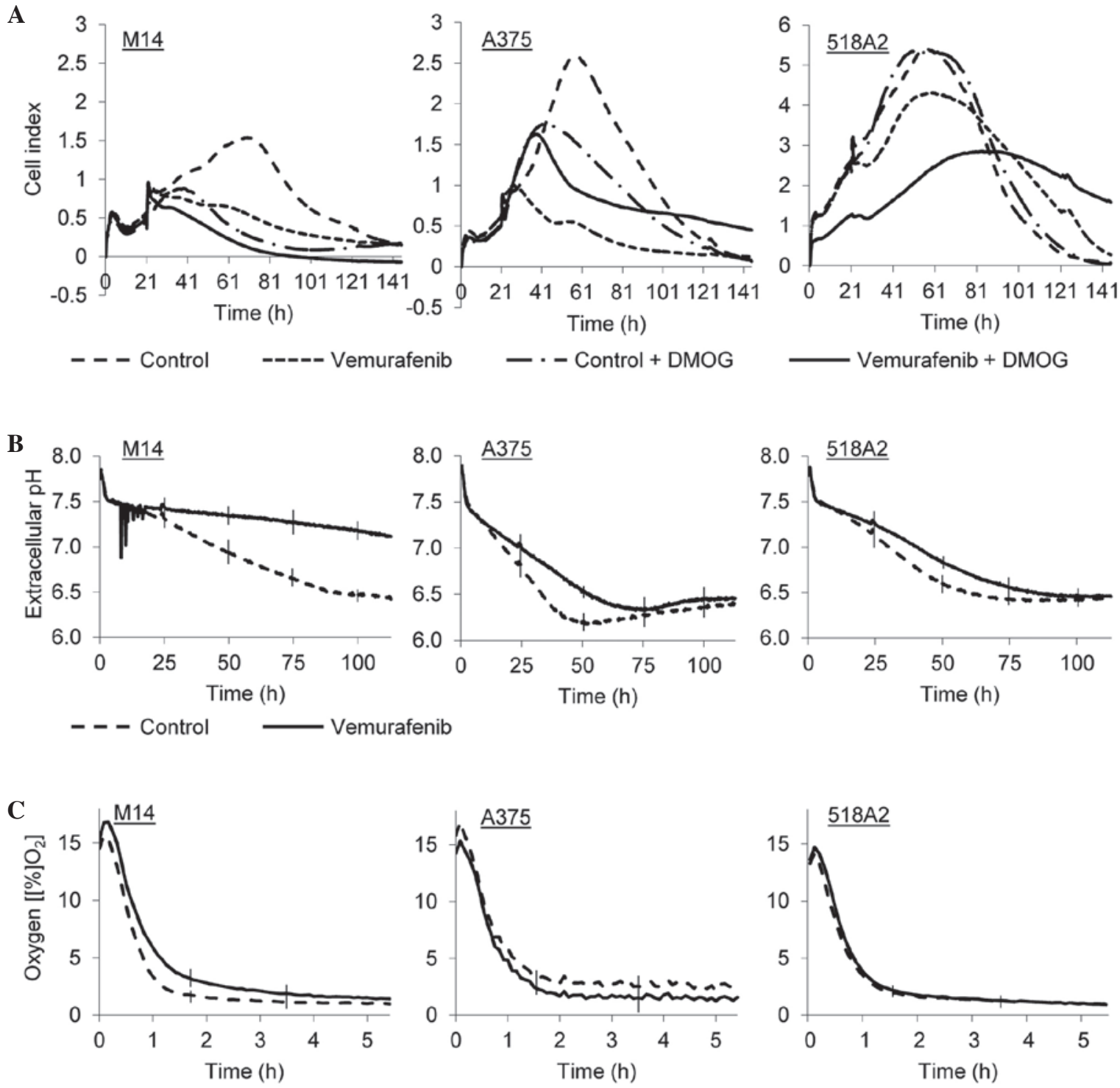

- - - Control Vemurafenib

Figure 1. Hypoxia influences the cell growth and the metabolism of melanoma cells following treatment with vemurafenib. (A) A time-course of the inhibitory effect of vemurafenib in melanoma cells under normoxic and hypoxic conditions. (B) A graph showing the effect of vemurafenib on extracellular pH in hypoxic melanoma cells. (C) A graph showing the effect of vemurafenib on oxygen consumption in hypoxic melanoma cells. The data are expressed as the mean \pm standard deviation of three independent experiments. DMOG, dimethyloxalylglycine.

in 518A2 cells. An increase in the expression levels of p-AKT and PKC $\alpha$ was observed in hypoxic A375 and 518A2 cells compared with normoxic cells. Exposure to hypoxia resulted in an upregulation of p-STAT3 in vemurafenib-treated A375 cells (Fig. 2C).

HIFla knockdown affects mitogen-activated protein kinase (MAPK) and phosphatidylinositol-4,5-bisphosphate 3-kinase (PI3K)/AKT pathway proteins in A375 cells. To assess whether knockdown of HIFl $\alpha$ affected signaling pathway proteins in A375 cells, an siRNA-based approach was used. This resulted in a significant reduction of HIFla (Fig. 3A). Additionally, the silencing of HIFla decreased the protein expression of CAIX (Fig. 3A) and affected the expression levels of numerous MAPK and PI3K/AKT pathway proteins. The knockdown of HIFl $\alpha$ decreased the expres- sion of p-AKT, which was restored following the treatment with vemurafenib. By contrast, the expression of p-ERK was increased following the knockdown of HIFl $\alpha$ and decreased following treatment with vemurafenib. No change was observed in the protein expression levels of p-FAK and PKC $a$ following the silencing of HIFla (Fig. 3B).

Hypoxia enhances cell migration of vemurafenib-treated A375 melanoma cells. To assess directional cell migration in vitro, a wound-healing assay was performed. Migration was induced in all three cell lines by hypoxia. When vemurafenib was added, hypoxic cells behaved differently. After $8 \mathrm{~h}, 518 \mathrm{~A} 2$ cells reduced the migration by $34 \%$, M14 cells revealed no changes and A375 cells increased migration by $20 \%$ compared with hypoxic untreated cells (Fig. 4). 
A
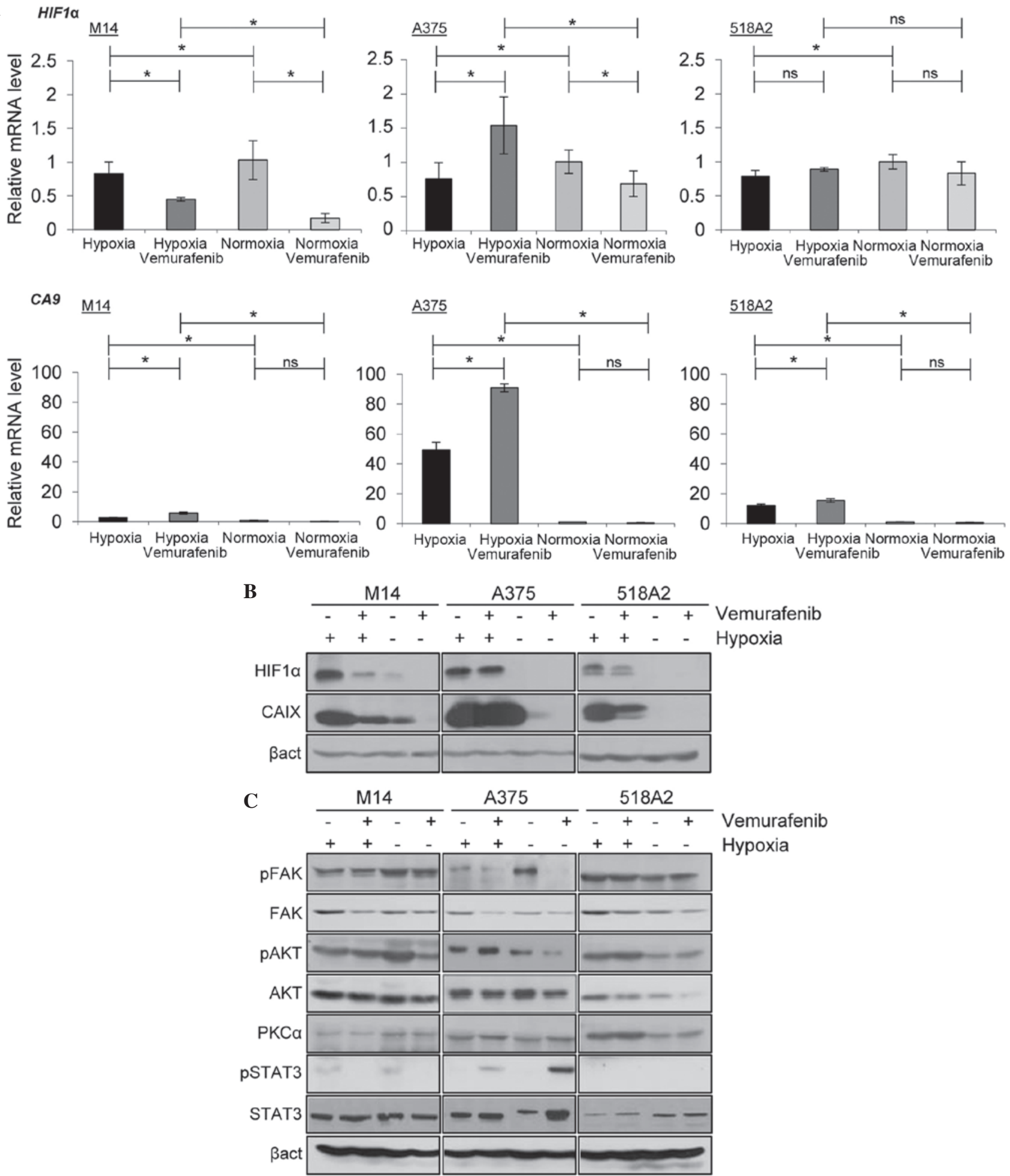

Figure 2. Effect of hypoxia on HIF1 $\alpha$, CAIX and multiple signaling pathway proteins in melanoma cells treated with vemurafenib. (A) Reverse transcription-quantitative polymerase chain reaction analysis of the mRNA expression levels of HIF1 $\alpha$ and CA9 normalized against $\beta$-act. The data are presented as the mean \pm standard deviation of three independent experiments ( ${ }^{*} \mathrm{P}<0.05$; ns, not significant). (B) The protein expression levels of HIF1 $\alpha$ and CAIX in vemurafenib-treated melanoma cells were assessed by immunoblotting. (C) Immunoblotting of proteins involved in the phosphatidylinositol-4,5-bisphosphate 3-kinase and Janus kinase-STAT pathways under normoxic and hypoxic conditions after vemurafenib treatment. STAT, signal transducer and activator of transcription; p-, phosphorylated; HIF, hypoxia-inducible factor; CA, carbonic anhydrase; act, actin; FAK, focal adhesion kinase; AKT, protein kinase B; PKC, protein kinase $\mathrm{C}$.

\section{Discussion}

The present study explored the role of hypoxia as a cause of melanoma heterogeneity. The characterization of different subsets of melanoma cells in a tumor lesion is of major clinical relevance for a successful therapeutic intervention $(15,20)$. Hypoxia not only promotes the growth of a tumor by enhancing glycolytic flux and increasing angiogenesis, 


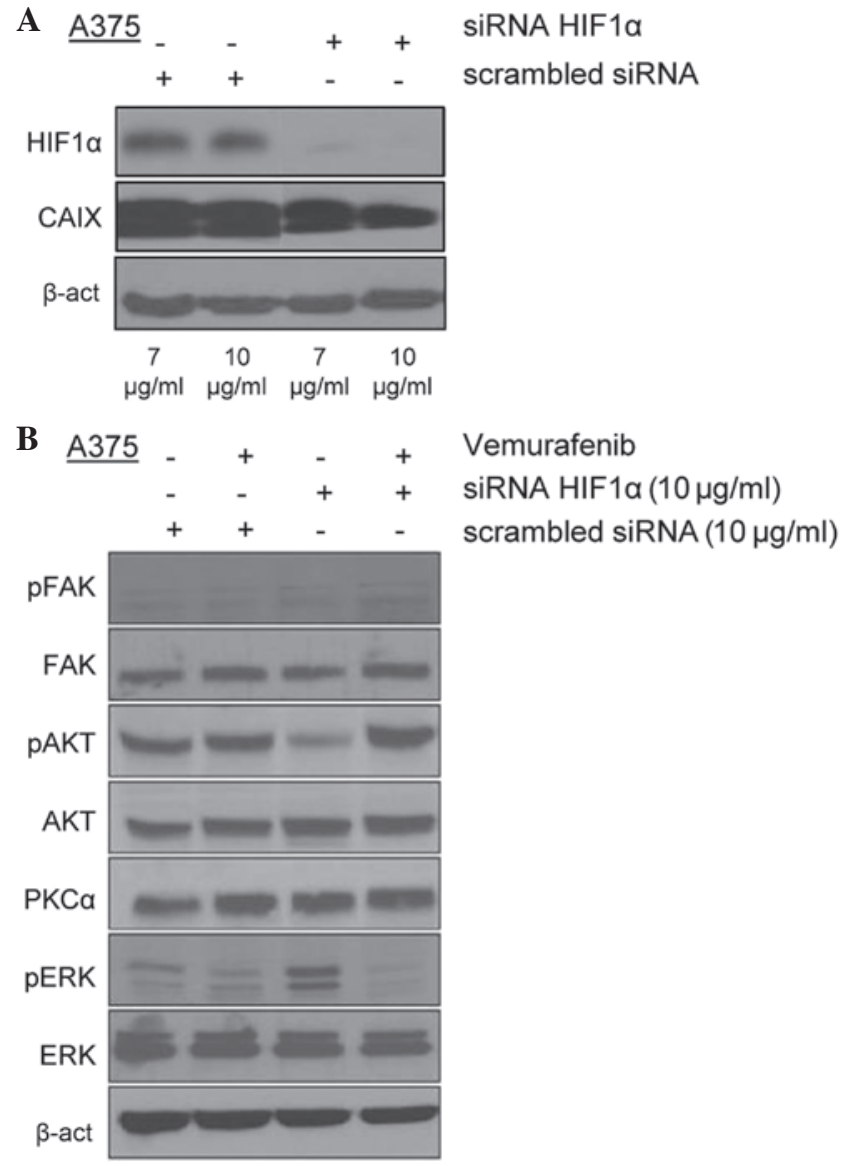

Figure 3. Effect of HIF1 $\alpha$ silencing on the expression of multiple signaling pathway proteins in A375 melanoma cells treated with vemurafenib. (A) Silencing of HIF1 $\alpha$ in A375 cells and its effects on the expression of CAIX. (B) Immunoblotting of proteins involved in the phosphatidylinositol-4,5-bisphosphate 3-kinase and mitogen-activated protein kinases pathways following silencing of HIF1 $\alpha$ in A375 cells after vemurafenib treatment. si, small interfering; p-, phosphorylated; HIF, hypoxia-inducible factor; $\mathrm{CA}$, carbonic anhydrase; act, actin; FAK, focal adhesion kinase; AKT, protein kinase B; PKC, protein kinase C; ERK, extracellular-signal-regulated kinases.

but also allows tumors to acquire resistance to anticancer drugs $(3,15)$. Vemurafenib is a specific inhibitor of the mutated BRAF-kinase and is used to treat patients with metastatic melanoma presenting the BRAF-V600 activating mutation (13). All cell lines harboring the BRAF-V600E-mutation tested were sensitive to vemurafenib under normoxic conditions in a real-time setting using the impedance-based xCELLigence system $^{\circledR}$ (Fig. 1A). However, a different proliferative response was observed when these cell lines were treated with vemurafenib in a hypoxic microenvironment. M14 and 518A2 cells continued to respond to the drug and decreased cell proliferation rates, whereas hypoxic, vemurafenib-treated A375 cells demonstrated an increase of cell proliferation rate by $20 \%$ compared with normoxic, vemurafenib-treated A375 cells (Fig. 1A). Hypoxia induces acidification of the tumor microenvironment, which promotes cell migration and invasion (2). Low pHe is a consequence of the abnormal metabolism in the tumor and a supportive factor for its progression (2). Hypoxic M14 cells displayed less pronounced extracellular acidosis, reaching a pHe value of $6.5 \pm 0.03$ following $90 \mathrm{~h}$ (Fig. 1B). Vemurafenib prevented the acidosis and caused a shift in $\mathrm{pHe}$ from acidic to alkaline for this cell line, suggesting that the
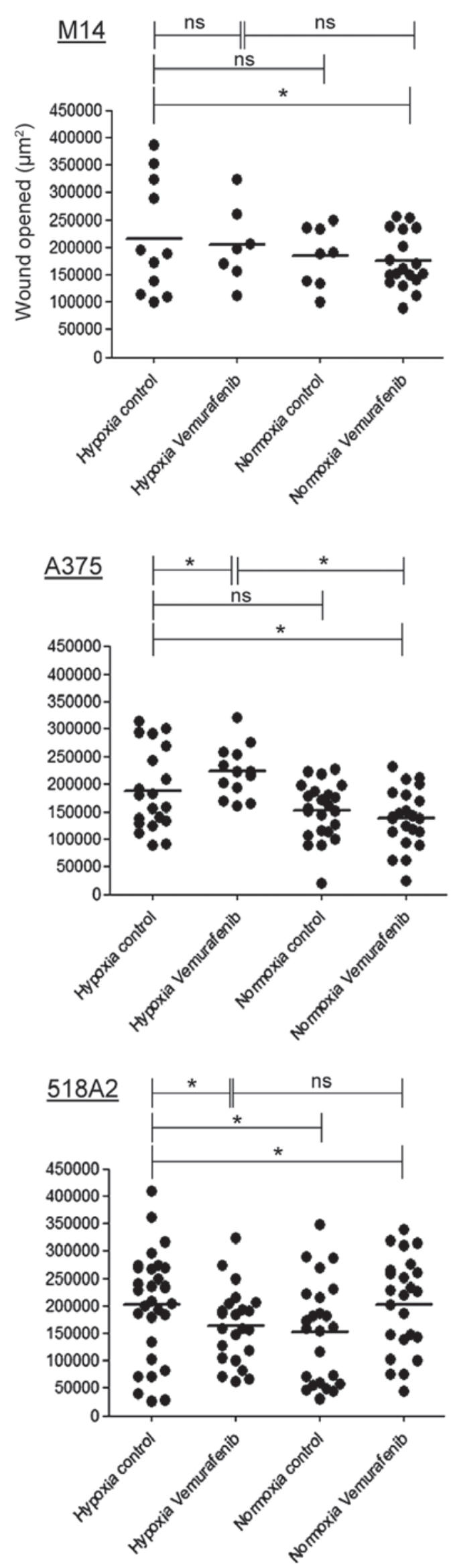

Figure 4. Hypoxia influences cell motility following vemurafenib treatment. The migration of melanoma cells treated with vemurafenib in normoxic and hypoxic conditions was determined by a wound-healing assay $($ " $\mathrm{P}<0.05$; ns, not significant). 
metabolism of the treated cells was significantly reduced. This was accompanied by decreased oxygen consumption (Fig. 1B and C). Altogether, this indicated that M14 cells retained their sensitivity to vemurafenib even under hypoxia. In hypoxic A375 and 518A2 cells, pHe values were rendered acidic $(<6.5)$ in a relatively short period of time. Shifts following vemurafenib treatment were smaller and reached $\mathrm{pHe}$ values comparable to untreated cells after $\sim 80 \mathrm{~h}$ (Fig. 1B). These data provided pathophysiological evidence of a diminished response to vemurafenib under hypoxia, which was further supported by a minor change in oxygen consumption of these cells (Fig. 1C). The survival of resistant A375 and 518A2 cell pools was also reflected in equalized pHe values, whereas the pHe for M14 cells was increased due to the rapid die-off of vemurafenib-sensitive M14 cells.

The main transcriptional regulator of the hypoxic response, HIF1, has been shown to be associated with melanomas (3). HIF1 serves an essential role in the maintenance of oxygen homeostasis and controls the expression of hundreds of genes, including CAIX, mediating developmental and physiological pathways. All melanoma cell lines tested expressed the hypoxic factors, HIF1 $\alpha$ and CAIX, and vemurafenib decreased their expression levels in hypoxic 518A2 and M14 cells (Fig. 2B). Notably, in hypoxic A375 cells, the protein expression levles of HIF1 $\alpha$ and CAIX remained unchanged following vemurafenib treatment (Fig. 2B), suggesting that vemurafenib had no affect on the hypoxic fraction of this cell line. This was not reflected in the mRNA expression levels of HIF1 $\alpha$ and CAIX, suggesting that the transcription of HIFl $\alpha$ and CAIX was not influenced by vemurafenib (Fig. 2A). It has been previously shown that the BRAF-V600E mutation increased HIF1 $\alpha$, suggesting that the expression of HIF1 $\alpha$ is partially regulated via the MAPK/ERK pathway (8).

The present study subsequently focused its attention on the effect of hypoxia and vemurafenib on proteins involved in the PI3K, MAPK and Janus kinase-STAT signaling pathways. It was determined that hypoxia and vemurafenib differently influenced signaling pathway proteins, including p-FAK, p-AKT, PKC $\alpha$ and p-STAT3, in the three cell lines. The expression of p-FAK was downregulated in hypoxic A375 and M14 cells, and was upregulated in hypoxic 518A 2 cells. An increase in the expression levels of p-AKT and PKC $\alpha$ was observed in hypoxic A375 and 518A2 cells. Hypoxia induced an upregulation in the expression of p-STAT3 in vemurafenib-treated A375 cells (Fig. 2C). Since the expression levels of HIF1 $\alpha$ and CAIX were not decreased in hypoxic A375 cells following treatment with vemurafenib, the present study silenced $H I F l \alpha$ using an siRNA-based approach (Fig. 3A) and evaluated the effects of silencing on the PI3K and MAPK signaling pathways. Silencing of $H I F l \alpha$ in hypoxic A375 cells restored the protein expression of p-ERK and the expression decreased again following treatment with vemurafenib. By contrast, the expression of p-AKT decreased after silencing of HIFl $\alpha$ and resumed following the treatment with vemurafenib (Fig. 3B). The data highlighted the complexity of the hypoxic melanoma phenotype and the challenge of optimizing BRAF-targeted therapy in this disease. Hypoxia was suggested as a possible facilitator of migration, switching between a proliferative to invasive phenotype in melanoma cells $(3,20)$. All three cell lines increased the migration under hypoxic conditions compared with normoxic conditions (Fig. 4). However, following treatment with vemurafenib, hypoxic A375 cells increased their migratory capacity, whereas hypoxic 518A2 cells exhibited reduced migration (Fig. 4).

The present findings supported the assumption that melanoma cell populations are not homogenous, however, consist of subpopulations with differing migrative, proliferative and tumor-initiating potentials. The development of resistance to vemurafenib can be explained, only in part, by the activation of other signaling pathways. A major challenge in cancer research is therefore to develop methods to characterize cell heterogeneity, and novel strategies to overcome resistance and target alternative pathways, which are not hampered by hypoxia.

\section{Acknowledgements}

The present study was a part of the EU Marie Curie Initial Training Network Biomedical engineering for cancer and brain disease diagnosis and therapy development (no. PITN-GA-2010-264417).

\section{References}

1. Rohwer $\mathrm{N}$ and Cramer $\mathrm{T}$ : Hypoxia-mediated drug resistance: Novel insights on the functional interaction of HIFs and cell death pathways. Drug Resist Update 14: 191-201, 2011.

2. Pastorek J and Pastorekova S: Hypoxia-induced carbonic anhydrase IX as a target for cancer therapy: From biology to clinical use. Semin Cancer Biol 31: 52-64, 2015.

3. Hanna SC, Krishnan B, Bailey ST, Moschos SJ, Kuan PF, Shimamura T, Osborne LD, Siegel MB, Duncan LM, O'Brien ET III, et al: HIF1 alpha and HIF2 $\alpha$ independently activate SRC to promote melanoma metastases. J Clin Invest 123: 2078-2093, 2013.

4. Semenza GL: Hypoxia-inducible factors: Mediators of cancer progression and targets for cancer therapy. Trends Pharmacol Sci 33: 207-214, 2012.

5. Ivanov S, Liao SY, Ivanova A, Danilkovitch-Miagkova A, Tarasova N, Weirich G, Merrill MJ, Proescholdt MA, Oldfield EH, Lee J, et al: Expression of hypoxia-inducible cell-surface transmembrane carbonic anhydrases in human cancer. Am J Pathol 158: 905-919, 2001.

6. Chrastina A, Závada J, Parkkila S, Kaluz T, Kaluzová M, Rajcáni J, Patorek J and Pastoreková S: Biodistribution and pharmacokinetics of I-125I-labeled monoclonal antibody M75 specific for carbonic anhydrase IX, an intrinsic marker of hypoxia, in nude mice xenografted with human colorectal carcinoma. Int J Cancer 105: 873-881, 2003.

7. Syrjänen L, Luukkaala T, Leppilampi M, Kallioinen M, Pastorekova S, Pastorek J, Waheed A, Sly WS, Parkkila S and Karttunen T: Expression of cancer-related carbonic anhydrases IX and XII in normal skin and skin neoplasms. APMIS 122: 880-889, 2014.

8. Kumar SM, Yu H, Edwards R, Chen L, Kazianis S, Brafford P, Acs G, Herlyn M and Xu XW: Mutant V600E BRAF increases hypoxia inducible factor-1alpha expression in melanoma. Cancer Res 67: 3177-3184, 2007.

9. Halaban R, Zhang W, Bacchiocchi A, Cheng E, Parisi F, Ariyan S, Krauthammer M, McCusker JP, Kluger Y and Sznol M: PLX4032, a selective BRAF (V600E) kinase inhibitor, activates the ERK pathway and enhances cell migration and proliferation of BRAF (WT) melanoma cells. Pigm Cell Melanoma Res 23: 190-200, 2010.

10. Botton T, Yeh I, Nelson T, Vemula SS, Sparatta A, Garrido MC, Allegra M, Rocchi S, Bahadoran P, McCalmont TH, et al: Recurrent BRAF kinase fusions in melanocytic tumors offer an opportunity for targeted therapy. Pigment Cell Melanoma Res 26: 845-851, 2013.

11. Azijli K, Stelloo E, Peters GJ and VAN DEN Eertwegh AJ: New developments in the treatment of metastatic melanoma: Immune checkpoint inhibitors and targeted therapies. Anticancer Res 34: 1493-1505, 2014. 
12. Lee JT, Li L, Brafford PA, van den Eijnden M, Halloran MB, Sproesser K, Haass NK, Smalley KS, Tsai J, Bollag G and Herlyn M: PLX4032, a potent inhibitor of the B-Raf V600E oncogene, selectively inhibits V600E-positive melanomas. Pigment Cell Melanoma Res 23: 820-827, 2010.

13. Chapman PB, Hauschild A, Robert C, Haanen JB, Ascierto P, Larkin J, Dummer R, Garbe C, Testori A, Maio M, et al: Improved survival with vemurafenib in melanoma with BRAF V600E mutation. N Engl J Med 364: 2507-2516, 2011.

14. Wagenaar TR, Ma LY, Roscoe B, Park SM, Bolon DN and Green MR: Resistance to vemurafenib resulting from a novel mutation in the BRAFV600E kinase domain. Pigm Cell Melanoma Res 27: 124-133, 2014

15. O'Connell MP, Marchbank K, Webster MR, Valiga AA, Kaur A, Vultur A, Li L, Herlyn M, Villanueva J, Liu Q, et al: Hypoxia induces phenotypic plasticity and therapy resistance in melanoma via the tyrosine kinase receptors ROR1 and ROR2. Cancer Discov 3: 1378-1393, 2013.

16. Wagner S, Hafner C, Allwardt D, Jasinska J, Ferrone S, Zielinski CC, Scheiner O, Wiedermann U, Pehamberger $\mathrm{H}$ and Breiteneder $\mathrm{H}$. Vaccination with a human high molecular weight melanoma-associated antigen mimotope induces a humoral response inhibiting melanoma cell growth in vitro. J Immunol 174: 976-982, 2005.
17. Pastoreková S, Závadová Z, Kostál M, Babusíková O and Závada J: A novel quasi-viral agent, Ma Tu, is a 2-component system. Virology 187: 620-626, 1992.

18. Svastova E, Witarski W, Csaderova L, Kosik I, Skvarkova L, Hulikova A, Zatovicova M, Barathova M, Kopacek J, Pastorek J and Pastorekova S: Carbonic anhydrase IX interacts with bicarbonate transporters in lamellipodia and increases cell migration via its catalytic domain. J Biol Chem 287: 3392-3402, 2012.

19. Schneider CA, Rasband WS and Eliceiri KW: NIH Image to ImageJ: 25 years of image analysis. Nat Methods 9: 671-5, 2012.

20. Widmer DS, Hoek KS, Cheng PF, Eichhoff OM, Biedermann T, Raaijmakers MI, Hemmi S, Dunnmer R and Levesque MP: Hypoxia contributes to melanoma heterogeneity by triggering HIF1 $\alpha$-dependent phenotype switching. J Invest Dermatol 133: 2436-2443, 2013. 